\title{
The relationship between major depressive disorder and glucose parameters: A cross-sectional study in a Chinese population
}

\author{
You-Fan Peng ${ }^{A-F}$, Shi-Mao Zhong ${ }^{B}$, Yu-Hua Qin ${ }^{B}$ \\ Department of Laboratory Medicine, Affiliated Hospital of Youjiang Medical University for Nationalities, Baise, Guangxi, China \\ A - research concept and design; B - collection and/or assembly of data; C - data analysis and interpretation; \\ $D$ - writing the article; $E$ - critical revision of the article; $F$ - final approval of article
}

\section{Address for correspondence \\ You-Fan Peng}

E-mail:youfanpeng7177@sina.com

Funding sources

None declared

\section{Conflict of interest}

None declared

Received on August 30, 2015

Revised on December 16, 2015

Accepted on May 05, 2016

DOI

10.17219/acem/63023

\section{Copyright}

Copyright by Author(s)

This is an article distributed under the terms of the

Creative Commons Attribution Non-Commercial License

(http://creativecommons.org/licenses/by-nc-nd/4.0/)

\section{Abstract}

Background. Depressive symptoms have been linked with insulin resistance in middle-aged and elderly populations. A strong relationship between peripheral insulin resistance and glucose homeostasis imbalance has been well established in previous studies. The role of serum fructosamine and fasting blood glucose (FBG) in elevating glucose homeostasis has been documented in the literature.

Objectives. The aim of the study was to examine the association of serum fructosamine and FBG with major depressive disorder (MDD).

Material and methods. The study analyzed the clinical characteristics and biochemical parameters of 305 patients with MDD and 312 healthy individuals.

Results. Serum concentrations of lipoprotein-cholesterol (HDL-C), total protein (TP) and creatinine (Cr) were found to be significantly different between the two groups. Serum fructosamine and fasting blood glucose (FBG) concentrations were high in patients with MDD compared with healthy individuals $(2.3 \pm 0.26$ vs. $2.1 \pm 0.27, p=0.018 ; 4.7 \pm 0.45$ vs. $4.5 \pm 0.45, p<0.001)$. The levels of serum fructosamine and FBG were also significantly higher in patients with MDD when all participants were stratified by gender. Age was found to be positively correlated with FBG, serum fructosamine and $\mathrm{Cr}(r=0.203, p<0.001$; $r=0.129, p=0.025 ; r=0.129, p=0.024)$, and negatively correlated with $\operatorname{TP}(r=-0.114, p=0.047)$ in patients with MDD. However, there were no correlations between age and $\mathrm{FBG}$, serum fructosamine or $\mathrm{Cr}$ in the healthy controls. In a multivariate logistic regression analysis, increased serum fructosamine and FBG concentrations were positively associated with MDD independently of age and gender, after adjustment for age and potential confounding factors (OR $=6.313, \mathrm{C} 195 \%: 2.953-13.393, \mathrm{p}<0.001 ; \mathrm{OR}=2.251$, $\mathrm{C} 195 \%$ : $1.464-3.462, p<0.001)$.

Conclusions. The study results suggest that increased serum fructosamine and FBG concentrations are associated with depressive conditions, which may influence glucose metabolism and impair glucose homeostasis in patients with MDD.

Key words: serum fructosamine, fasting blood glucose, major depressive disorder 
A large body of epidemiologic data indicates that depressive conditions severely influence human physical health. Data show that inflammatory cytokines are considered to play a central role in mediating depression by communicating peripheral inflammation to the brain. ${ }^{1}$ Growing evidence has underlined an association between psychosocial factors and cardiovascular disease (CVD). ${ }^{2}$ Unfortunately, there is a consensus that depression may contribute to the clinical outcomes in several chronic diseases, such as diabetes, chronic kidney diseases (CKD) and CVD. ${ }^{2,3}$ Very recently, a noteworthy prevalence of metabolic syndrome (MS) was observed in patients with depression. ${ }^{3}$

Poor blood glucose homeostatic regulation in the body may result in adverse clinical outcomes. Serum fructosamine is a glycoprotein formed through a nonenzymatic mechanism, and it is clinically used to estimate glycemic control over the previous two to three weeks in patients with diabetes. ${ }^{4}$ Earlier investigations have shown that serum concentrations of fructosamine are negatively correlated with estimated glomerular filtration rate (GFR) in non-diabetic individuals, and are associated with microvascular complications and retinopathy in both diabetic and non-diabetic individuals. ${ }^{5-8}$ Fasting blood glucose (FBG) is closely associated with reduced GFR in elderly healthy Chinese patients, and elevated FBG at admission is a predictor of all-cause mortality in patients with dilated cardiomyopathy. ${ }^{9,10}$ Interestingly, depressive symptoms have been linked with insulin resistance in middleaged and elderly populations. ${ }^{11}$ Rafacho et al. suggested a strong relationship between peripheral insulin resistance and glucose homeostasis imbalance. ${ }^{12}$ The role of serum fructosamine and FBG in elevated glucose homeostasis has been documented in the literature. ${ }^{13}$ Therefore, the present study examined the association of serum fructosamine and FBG with major depressive disorder (MDD).

\section{Material and methods}

This study retrospectively analyzed demographic data and biochemical parameters in 305 patients with MDD and 312 healthy individuals. The average age was $36.8 \pm 9.70$ years in all the participants, $39.9 \pm 8.47$ years in the patients with MDD and $33.8 \pm 9.86$ among the healthy individuals. The diagnosis of MDD was established according to DSM-IV criteria. ${ }^{14,15}$ The study complied with all the relevant national regulations, institutional policies and in accordance the tenets of the Helsinki Declaration regarding research involving humans, and was approved by the Ethics Committee of the Affiliated Hospital of Youjiang Medical University for Nationalities (Baise, Guangxi, China).

Criteria for exclusion from the present study included other primary psychiatric disorders, diabetes mellitus, hypertension, CVD, endocrine diseases, MS, liver or kid- ney dysfunction, pregnancy, acute or chronic infections, malignant tumors, immune diseases, a history of trauma, and the use of psychiatric medications.

Fasting venous blood was collected from all the participants, and the values of fructosamine, low density lipoprotein-cholesterol (LDL-C), total cholesterol (TC), high density lipoprotein-cholesterol (HDL-C), fasting blood glucose (FBG), total protein (TP), triglyceride (TG) and creatinine $(\mathrm{Cr})$ were measured using automated biochemical analysis (Cobas 8000 modular analyzer, Hoffman-La Roche AG, Basel, Switzerland ).

\section{Statistical analysis}

SPSS 16.0 software (SPSS Inc., Chicago, USA) was used to analyze the data. Continuous variables were presented as mean \pm standard deviation. The Kolmogorov-Smirnov test was used to examine the normality of the data. Student's t-test, the chi ${ }^{2}$ test and the Mann-Whitney $U$ test were used to compare the baseline information, and Student's t-test was used to compare the levels of serum fructosamine and FBG in male and female MDD patients with those of healthy males and females. To further assess the association of age with biochemical parameters, correlations between age and biochemical parameters were checked using the Pearson approach. Binary logistic regression analysis was also used to identify the underlying factors associated with MDD. A $p$ value $<0.05$ was regarded as statistically significant.

\section{Results}

Cumulative results for all the parameters were analyzed in 238 males and 379 females. The laboratory parameters and demographic characteristics of the participants are shown in Table 1 . In the baseline data, the serum concentrations of HDL-C, TP and $\mathrm{Cr}$ were found to be significantly different between the two groups. Notably, serum fructosamine and FBG concentrations were significantly higher in the patients with MDD than in the healthy individuals. A decision was made to separate the study population into genders to compare the differences in serum fructosamine and FBG concentrations. Since depression has a higher prevalence in females than males, when all the individuals were stratified by gender the results showed that the serum concentrations of fructosamine and FBG were also significantly higher in the patients with MDD compared with the healthy subjects (Fig. 1 and 2).

Knowing that some biochemical parameters, such as FBG, fructosamine and lipids may be influenced by age, a linear analysis was performed for both the MDD patients and the healthy controls; the results indicated that age was positively correlated with TC, LDL-C, TG in 
Table 1. Laboratory parameters and demographic characteristics in patients with major depressive disorder and healthy individuals

\begin{tabular}{|c|c|c|c|}
\hline \multirow{2}{*}{ MDD patients } & \multicolumn{3}{|c|}{ Controls } \\
\hline & $n=305$ & $n=312$ & $p$-value \\
\hline Gender (male/female) & $66 / 239$ & $172 / 140$ & $<0.001$ \\
\hline Age(y) & $39.9 \pm 8.47$ & $33.8 \pm 9.86$ & $<0.001$ \\
\hline Body mass index $\left(\mathrm{kg} / \mathrm{m}^{2}\right)$ & $24.0 \pm 2.63$ & $24.1 \pm 3.01$ & 0.852 \\
\hline Total cholesterol (mmol/L) & $4.2 \pm 0.75$ & $4.1 \pm 0.81$ & 0.346 \\
\hline $\begin{array}{l}\text { Low density lipoprotein } \\
\text { cholesterol (mmol/L) }\end{array}$ & $2.6 \pm 0.63$ & $2.5 \pm 0.68$ & 0.066 \\
\hline $\begin{array}{l}\text { High density lipoprotein } \\
\text { cholesterol (mmol/L) }\end{array}$ & $1.3 \pm 0.34$ & $1.2 \pm 0.33$ & 0.002 \\
\hline $\begin{array}{l}\text { Fasting blood-glucose } \\
(\mathrm{mmol} / \mathrm{L})\end{array}$ & $4.7 \pm 0.45$ & $4.5 \pm 0.45$ & $<0.001$ \\
\hline Total protein (g/L) & $66.8 \pm 5.05$ & $69.9 \pm 5.20$ & $<0.001$ \\
\hline Triglycerides (mmol/L) & $1.0 \pm 0.34$ & $1.00 \pm 0.34$ & 0.202 \\
\hline Creatinine (mmol/L) & $58.2 \pm 12.47$ & $64.3 \pm 13.56$ & $<0.001$ \\
\hline Serum fructosamine (mmol/L) & $2.3 \pm 0.26$ & $2.1 \pm 0.27$ & 0.018 \\
\hline
\end{tabular}

both the MDD patients and the healthy individuals. Interestingly, age was found to be positively correlated with FBG, serum fructosamine and $\mathrm{Cr}(\mathrm{r}=0.203$, $\mathrm{p}<0.001$; $\mathrm{r}=0.129, \mathrm{p}=0.025 ; \mathrm{r}=0.129, \mathrm{p}=0.024)$ and negatively correlated with TP $(\mathrm{r}=-0.114, \mathrm{p}=0.047)$ in the patients with MDD. However, there were no correlations between age and FBG, serum fructosamine or $\mathrm{Cr}$ in the control group. Potential confounding factors, including age, were therefore adjusted in the multivariate logistic regression analysis. The results of multiple logistic regression analyses revealed that increased serum fructosamine and FBG concentrations were independently associated with MDD (Table 2).

\section{Discussion}

In the present study, a statistically significant association was found between elevated serum fructosamine, FBG concentrations and MDD in multiple logistic regression analyses, and a higher prevalence of MDD was observed in elderly females.

Depressive conditions have been recognized to be a risk factor for the development and progression of some chronic diseases, especially for cardiovascular disease. In earlier trials, several longitudinal studies reported an association between depression and coronary heart disease in patients with diabetes. ${ }^{16,17}$ It has recently been highlighted that depression increases the risk of CVD in
Table 2. Serum fructosamine and fasting blood glucose associated with major depressive disorder in the multivariate regression analysis

\begin{tabular}{|l|c|c|c|}
\multicolumn{1}{|c|}{ Parameters } & OR & Cl 95\% & p-value \\
\hline Gender & 0.142 & $0.092-0.220$ & $<0.001$ \\
$\begin{array}{l}\text { Age } \\
\text { (years) }\end{array}$ & 1.059 & $1.037-1.082$ & $<0.001$ \\
$\begin{array}{l}\text { Fasting blood-glucose } \\
\text { (mmol/L) }\end{array}$ & 2.251 & $1.464-3.462$ & $<0.001$ \\
$\begin{array}{l}\text { Serum fructosamine } \\
\text { (mmol/L) }\end{array}$ & 6.313 & $2.953-13.393$ & $<0.001$ \\
\hline
\end{tabular}

Fig. 1. Increased serum fructosamine concentrations in MDD patients were shown when the study participants were stratified by gender

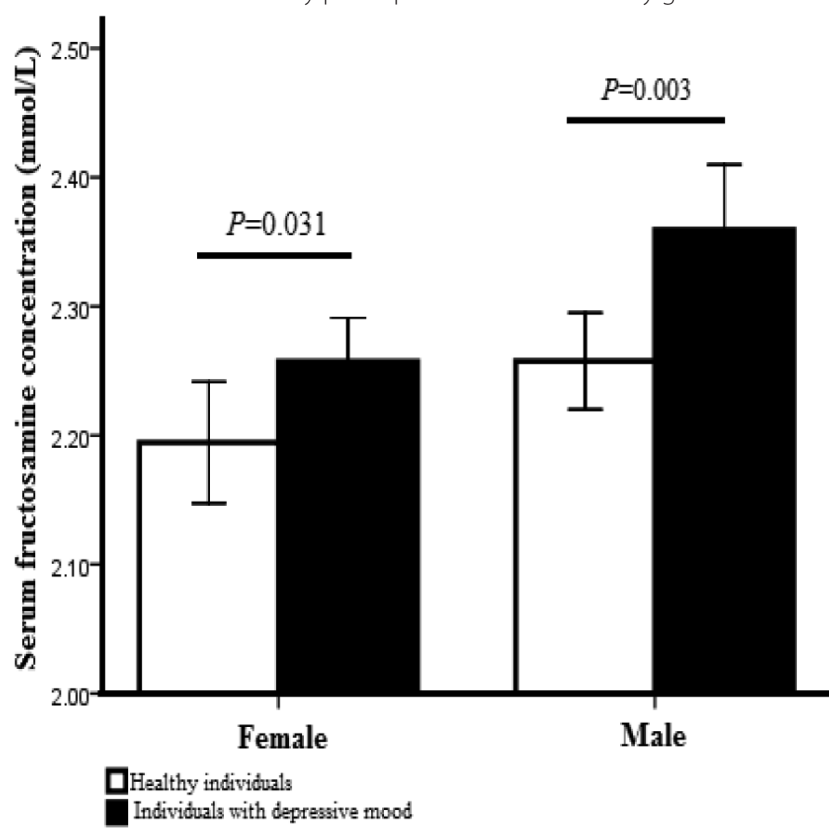

Fig. 2. Increased fasting blood glucose concentrations in MDD patients were shown when the study participants were stratified by gender

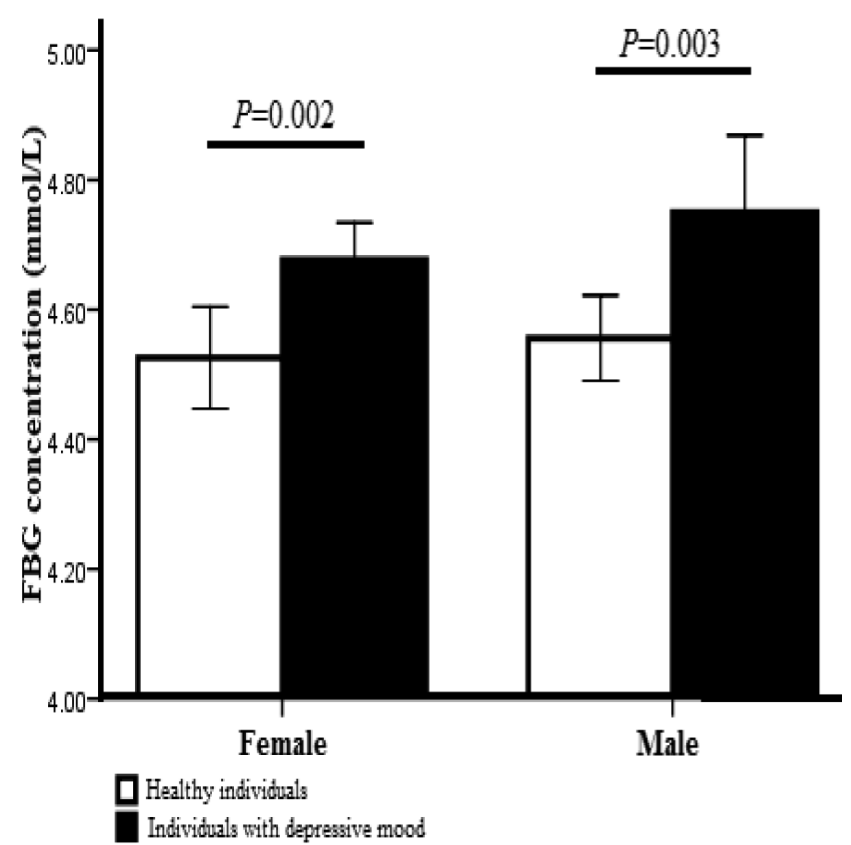


the presence or absence of heart disease. ${ }^{18}$ The association between depression and future coronary heart disease (CHD) has been a noteworthy research topic. ${ }^{18}$ In the present study, increased fructosamine and FBG concentrations were observed in the patients with MDD. Several possible interpretations can be put forward to explain the reasons for high levels of fructosamine and FBG in patients with depression. First, oxidative stress and inflammatory factors in MDD patients have been considered an underlying mechanism explaining the relationship between depressive conditions and cardiovascular disease. ${ }^{19}$ Importantly, there is now growing evidence linking oxidative stress to insulin resistance through direct and indirect mechanisms, and the inflammatory cascades have also been associated with increased insulin resistance. ${ }^{20,21}$ On the other hand, depressive conditions can suppress hyperactivity of the hypothalamicpituitary-adrenocortical (HPA) axis, which is a risk factor for insulin resistance. ${ }^{22}$ Moreover, serotonin dysfunction in the nervous system has been reported in patients with depression, and selective serotonin re-uptake inhibitors used as antidepressant agents can improve insulin sensitivity independently of body weight. ${ }^{23}$ Remarkably, fasting insulin concentrations are significantly higher in patients with depressive symptoms compared with those without depressive symptoms. ${ }^{11}$ Pan et al. also observed a positive correlation between depressive symptoms and insulin resistance among middle-aged and elderly Chinese patients. ${ }^{11}$ Clearly, a depressive condition may increase insulin resistance in MDD patients without diabetes. ${ }^{24}$

Insulin resistance plays a crucial role in the pathogenesis of diabetes. It has been suggested that common glycemic load measurements, such as postload glucose, FBG and fructosamine, are strongly associated with the risk of diabetes. ${ }^{25,26}$ Previous studies have indicated that serum fructosamine levels are positively correlated with increased insulin resistance. ${ }^{25}$ In addition, it is well known that insulin resistance increases FBG levels in both diabetics and the non-diabetic population. Thus, increased fructosamine and FBG levels may be induced by insulin resistance in patients with $\mathrm{MDD}$, and the relationship may influence glucose metabolism and impair glucose homeostasis.

In the present study, lipid parameters were measured, which assessed the nutritional status of all the participants. Among these lipid parameters, higher levels of HDL-C have been reported to be associated with worse moods and higher levels of depression and anxiety. ${ }^{27}$ Those results were consistent with the trend toward high HDL-C values in the MDD patients in the present study. Interestingly, in the baseline information low TP and Cr were noted in the MDD patients compared with the healthy individuals, although there were no statistically significant differences in the multivariate logistic regression analysis. In malnutrition states, the low $\mathrm{TP}$ and $\mathrm{Cr}$ levels in MDD patients may be attributed to anorexia nervosa in patients with MDD. Accompanying anorexia nervosa is prevalent among MDD patients, and MDD is a psychiatric condition often associated with dietary restrictions, appetite disturbances and a less healthy diet, which may contribute to low TP and $\mathrm{Cr}$ in patients with MDD. ${ }^{28,29}$

Several limitations of the present study should be noted. Firstly, both glycosylated hemoglobin and insulin resistance levels in patients with MDD were missing from the results. Secondly, the study did not discuss other psychological and environmental variables that may influence the relationship between glucose parameters and depression. In addition, accompanying anorexia nervosa in MDD patients should have been considered a confounder in the current study. However, the data suggest that increased serum fructosamine and FBG concentrations are associated with depressive conditions, which may influence glucose metabolism and impair glucose homeostasis in patients with MDD.

\section{References}

1. Kent S, Bluthe RM, Dantzer R, Hardwick AJ, Kelley KW, Rothwell NJ. Different receptor mechanisms mediate the pyrogenic and behavioraleffects of interleukin-1. Proc Natl Acad Sci USA.1992;89:9117-9120.

2. Sararoodi BR. Type D penonality. J Res Behav Sci. 2009;7:75-86.

3. Topić R, Miličić D, Štimac Z, et al. Somatic comorbidity, metabolic syndrome, cardiovascular risk, and CRP in patients with recurrent depressive disorders. Croat Med J. 2013;54:453-459.

4. Armbruster DA. Fructosamine: structure, analysis, and clinical usefulness. Clin Chem. 1987;33:2153e63.

5. Peng YF, Cao WY, Zhao JM, et al. Association between serum fructosamine and kidney function in nondiabetic individuals without chronic kidney disease. Med Sci Monit. 2015;21:1996-1999.

6. Selvin E, Francis LM, Ballantyne CM, et al. Nontraditional markers of glycemia: associations with microvascular conditions. Diabetes Care. 2011;34:960e7.

7. Browner WS, Pressman AR, Lui LY, Cummings SR. Association between serum fructosamine and mortality in elderly women: the study of osteoporotic fractures. Am J Epidemiol. 1999;149:471e5.

8. Selvin E, Rawlings AM, Grams M, et al. Fructosamine and glycated albumin for risk stratification and prediction of incident diabetes and microvascular complications: a prospective cohort analysis of the Atherosclerosis Risk in Communities (ARIC) study. Lancet Diabetes Endocrinol. 2014;2:279e88.

9. Sun Y, Wang C, Yang W, et al. Fasting blood glucose, but not 2-h postload blood glucose or $\mathrm{HbA1c}$, is associated with mild decline in estimated glomerular filtration rate in healthy Chinese. Int Urol Nephrol. 2015;47(1):147-152.

10. Li X, Jiang R, Kong H, Shu Y, Li Q, Hua W. Fasting blood glucose at admission and survival in patients with dilated cardiomyopathy: a single-center cohort study. Exp Clin Endocrinol Diabetes. 2014;122(8):457-462.

11. Pan $A, Y e X$, Franco $O H$, et al. Insulin resistance and depressive symptoms in middle-aged and elderly Chinese: Findings from the nutrition and health of aging population in China Study. J Affect Disord. 2008;109(1-2):75-82.

12. Rafacho A, Ortsäter H, Nadal A, Quesada I. Glucocorticoid treatment and endocrine pancreas function: implications for glucose homeostasis, insulin resistance and diabetes. J Endocrinol. 2014;223(3):R49-62.

13. Tuomainen TP, Nyyssönen K, Salonen R, et al. Body iron stores are associated with serum insulin and blood glucose concentrations. Population study in 1,013 eastern Finnish men. Diabetes Care. 1997;20(3):426-428.

14. American Psychiatric Association. Diagnostic and statistical manual of mental disorders, fourth edition. 4th edn. American Psychiatric Association: Washington, DC, 2001. 
15. Wittchen HU. Reliability and validity studies of the WHO-Composite International Diagnostic Interview (CIDI): a critical review. J Psychiatr Res. 1994;28:57-84.

16. Black S, Markides K, Ray L. Depression predicts increased incidence of adverse death outcomes in older Mexican-Americans with type 2 diabetes. Diabetes Care. 2003;26:2822-2828.

17. Kinder L, Kamarck T, Baum A, Orchard T. Depressive symptomatology and coronary heart disease in type 1 diabetes mellitus. A study of possible mechanisms. Health Psychol. 2002;21: 542-552.

18. Wayne J. Katon, Elizabeth HB. Cardiac risk factors in patients with diabetes mellitus and major depression. J Gen Intern Med. 200;19:1192-1199.

19. Steptoe A. Depression and the development of coronary heart disease. In: Steptoe A, editor. Depression and physical illness. Cambridge: Cambridge University Press 2007: 53-86.

20. Keane KN, Cruzat VF, Carlessi R, de Bittencourt PI Jr, Newsholme P. Molecular events linking oxidative stress and inflammation to insulin resistance and $\beta$-cell dysfunction. Oxid Med Cell Longev. 2015;2015:181643.

21. Liu S, Tinker L, Song Y, Rifai N, Bonds DE. A prospective study of inflammatory cytokines and diabetes mellitus in a multiethnic cohort of postmenopausal women. Arch Intern Med. 2007;167:1676-1685.

22. Chan O, Inouye K, Riddell MC, Vranic M, Matthews SG. Diabetes and the hypothalamo-pituitary-adrenal (HPA) axis. Minerva Endocrinol. 2003;28:87-102.
23. Breum L, Bjerre U, Bak JF, Jacobsen S, Astrup A. Longterm effects of fluoxetine on glycemic control in obese patients with non-insulin-dependent diabetes mellitus or glucose intolerance: Influence on muscle glycogen synthase and insulin receptor kinase activity. Metabolism. 1995;44:1570-1576.

24. Selvin E, Rawlings AM, Grams M, Klein R, Sharrett AR, Steffes M, Coresh J. Fructosamine and glycated albumin for risk stratification and prediction of incident diabetes and microvascular complications: a prospective cohort analysis of the Atherosclerosis Risk in Communities (ARIC) study. Lancet Diabetes Endocrinol. 2014;2(4):279-288.

25. Zaccardi F1, Kurl S, Pitocco D, Ronkainen K, Laukkanen JA. Serum fructosamine and risk of type 2 diabetes mellitus among middleage Finnish men: A 23-year population-based prospective study. Acta Diabetol. 2015;52(1):161-166.

26. Rathmann W, Kowall B, Heier $M$, et al. Prediction models for incident type 2 diabetes mellitus in the older population: A cohort study. Diabet Med. 2010;27(10):1116-1123.

27. Lieberman HR, Kellogg MD, Kramer FM, Bathalon GP, Lesher LL. Lipid and other plasma markers are associated with anxiety, depression, and fatigue. Health Psychol. 2012;31(2):210-216.

28. Brand-Gothelf A, Leor S, Apter A, Fennig S. The impact of comorbid depressive and anxiety disorders on severity of anorexia nervosa in adolescent girls. J Nerv Ment Dis. 2014;202(10):759-762.

29. Whited MC, Schneider KL, Appelhans BM, et al. Severity of depressive symptoms and accuracy of dietary reporting among obese women with major depressive disorder seeking weight loss treatment. PLoS One. 2014;9(2):e90361. 